\title{
Akuter Schwindel - Herausforderung für den erstbehandelnden Arzt
}

\author{
Wettstein, V G
}

DOI: https://doi.org/10.1024/1661-8157/a002626

Other titles: Acute vertigo - a challenge for the emergency doctor

Posted at the Zurich Open Repository and Archive, University of Zurich ZORA URL: https://doi.org/10.5167/uzh-147164

Journal Article

Accepted Version

Originally published at:

Wettstein, V G (2017). Akuter Schwindel - Herausforderung für den erstbehandelnden Arzt. Praxis, 106(6):323-325.

DOI: https://doi.org/10.1024/1661-8157/a002626 


\section{Praxis}

Schweizerische Rundschau für Medizin

Revue Suisse de la médecine
(4) hogrefe

Jahrgang 106 / Heft 6

15. März 2017

Gelistet in MEDLINE, EMBASE und Scopus

\section{Herausgeber}

Edouard Battegay

Johann Steurer

Bernard Waeber

In Zusammenarbeit mit

Cochrane Schweiz

Swiss Young Internists

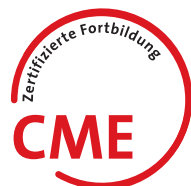

Antikörpermangelsyndrome

Neue Herzinsuffizienz-Guidelines: Update

WHO-Klassifikation der HPV-assoziierten

Zervix-Läsionen

Patienten mit psychisch bedingten Arbeitsproblemen

Radiuskopffraktur: Nicht immer unkompliziert

Akuter Schwindel

\section{Das Referenzwerk \\ der kindlichen Epilepsien}

Axel Panzer Tilman Polster Hartmut Siemes

Epilepsien bei Kindern und Jugendlichen

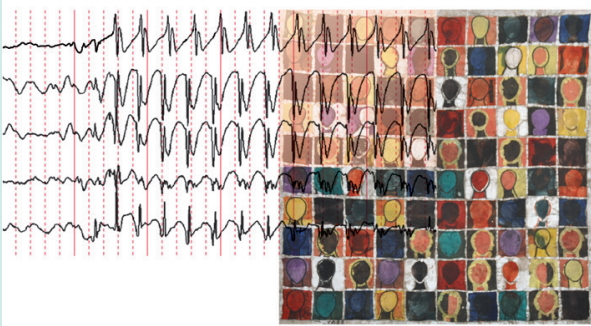

HUBER H
Axel Panzer / Tilman Polster / Hartmut Siemes

\section{Epilepsien bei Kindern und Jugendlichen}

3., vollst. überarb. u. erw. Aufl. 2015.

652 S., 26 Abb., 183 Tab., Gb

$€ 79,95$ / CHF 99.00

ISBN 978-3-456-85513-4

Auch als eBook erhältlich

Dieses Buch stellt allen denen, welche Kinder und Jugendliche mit epileptischen Anfällen oder Epilepsien medizinisch betreuen, umfassende aktuelle Informationen zur Verfügung. 


\section{Inhalt}

Editorial

Radiuskopffraktur - wie weiter?

Florin Allemann

Spezialsprechstunde Obere Extremitäten/Sportverletzungen,

Klinik für Unfallchirurgie, Universitätsspital Zürich

Continuing Medical

Education

\section{Antikörpermangelsyndrome}

287

Kristyna Valkova und Florence Vallelian

Klinik und Poliklinik für Innere Medizin, Universitätsspital Zürich

CME-Rheumatologie 11: Sternoclaviculäre Schwellung - immer ein 295 Tietze-Syndrom?

${ }^{1}$ Giorgio Tamborrini, ${ }^{2}$ Christoph Gut, ${ }^{3}$ Dirk Johannes Schaefer und

${ }^{4}$ Didier Lardinois

Ultraschall Zentrum Rheumatologie, Basel'; Rheumatologische Praxis, Reinach²; Plastische, Rekonstruktive, Ästhetische und Handchirurgie, Universitätsspital Basel, Universität Basel ${ }^{3}$; Thoraxchirurgie, Universitätsspital Basel, Universität Basel4

CME-Antworten zu den Fragen zu «Brugada-Syndrom» aus Praxis Nr. 5

${ }^{1}$ Danaë Parianos und ${ }^{2}$ Ardan M. Saguner

Klinik für Innere Medizin', Klinik für Kardiologie, Abteilung Rhythmologie², Universitätsspital Zürich

CME-Labor 49/Auflösung: Therapeutisches Drug-Monitoring

Daniel Müller

Institut für Klinische Chemie, Universitätsspital Zürich

Mini-Review

Neue Herzinsuffizienz-Guidelines: Update für die Praxis

297

New Heart Failure Guidelines: Update for Primary Care

Peter Rickenbacher

Abteilung für Kardiologie, Medizinische Universitätsklinik, Kantonsspital

Baselland, Bruderholz

News in der aktuell gültigen WHO-Klassifikation der HPV-assoziierten Zervix-Läsionen

News in the Current WHO Classification of the Cervical HPV-Associated Lesions

Beata Bode-Lesniewska und Rosmarie Caduff

Institut für Pathologie und Molakularpathologie, Universitätsspital Zürich

Patienten mit psychisch bedingten Arbeitsproblemen: Besonderheiten und Handlungsmöglichkeiten

Patients with Mental Health-related Problems at Work: Characteristics and Possible Interventions

Niklas Baer

Fachstelle Psychiatrische Rehabilitation, Psychiatrie Baselland, Liestal 
Not That Simple a Radial Head Fracture...

1,2,3 Patrick Vavken

alphaclinic Zürich'; Division of Sports Medicine, Children's Hospital Boston, Harvard Medical School, Boston, USA2; Center for Population and Development Studies, Harvard School of Public Health, Cambridge, USA ${ }^{3}$

Klinische Kurzstandards

Akuter Schwindel - Herausforderung für den erstbehandelnden Arzt

Acute Vertigo - a Challenge for the Emergency Doctor

Vincent G. Wettstein

Klinik für Ohren-, Nasen-, Hals- und Gesichtschirurgie, Interdisziplinäres

Zentrum für Schwindel und neurologische Sehstörungen, Universitätsspital

Zürich

Journal Club

Präventiver Effekt der Antikoagulanzien nach Knie- oder Hüftgelenksersatz: Risiko venöser Thromboembolien sinkt, Blutungsrisiko steigt Johann Steurer

Horten-Zentrum für praxisorientierte Forschung und Wissenstransfer, Universitätsspital Zürich

Neuroleptika haben keinen Platz in der Behandlung eines Delirs bei Palliativpatienten

Leander Muheim

Institut für Hausarztmedizin, Horten-Zentrum für praxisorientierte Forschung und Wissenstransfer, Universitätsspital Zürich 


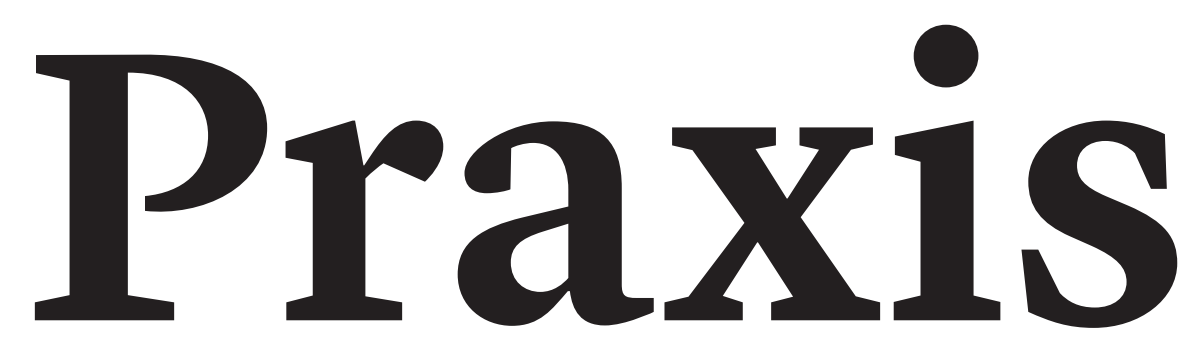

\section{Schweizerische Rundschau für Medizin Revue Suisse de la médecine}

Ihr Artikel wurde in einer Zeitschrift des Hogrefe Verlages veröffentlicht. Wir freuen uns, diesen e-Sonderdruck der veröffentlichten Verlagsfassung für den persönlichen Gebrauch der Autoren, zur Weiterleitung an die Koautoren sowie zur Verwendung auf individuelle Nachfrage für private Zwecke und als Teil eines Forschungsantrages oder im Rahmen einer Abschluss- oder Doktorarbeit zur Verfügung zu stellen.

Sofern Sie Ihren Artikel auf einer persönlichen oder institutionellen Webseite, in einem institutionellen oder disziplinären Repositorium oder in wissenschaftlichen Netzwerken (scholarly communication networks, SCNs) hinterlegen möchten, verwenden Sie bitte dieeingereichte Manuskriptfassung (vor peer review) oder dieakzeptierte Manuskriptfassung (nach peer review) entsprechend den Richtlinien der Publikationsfreigabe für Ihren Artikel sowie den Nutzungsrichtlinien für Hogrefe-Zeitschriftenartikel (http://hgf.io/ nutzungsrichtlinien). 


\title{
Akuter Schwindel - Herausforderung für den erstbe- handelnden Arzt
}

Dies ist eine Guideline, die für die Anwendung innerhalb der ORL-Klinik des Universitätsspitals Zürich erstellt

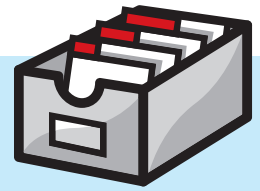
wurde. Die Guidelines haben in Qualitätszirkeln einen Reviewprozess durchlaufen und wurden für «Praxis» zusätzlich einem externen Reviewing unterzogen.

Sollten Sie andere Erfahrungen gemacht haben als hier berichtet oder anderer Meinung sein, schreiben Sie uns dies unter redaktion.praxis@hogrefe.ch.

\section{Acute Vertigo - a Challenge for the Emergency Doctor}

\author{
Vincent G. Wettstein
}

Klinik für Ohren-, Nasen-, Hals- und Gesichtschirurgie, Interdisziplinäres Zentrum für Schwindel und neurologische Sehstörungen, Universitätsspital Zürich

\section{Fragestellung}

Schwindel und Gleichgewichtsstörungen treten im Erwachsenenalter häufig auf. Verschiedene Ursachen können solche Beschwerden hervorrufen. Ein neuartiges, akut auftretendes Schwindelereignis ist für den Betroffenen meist bedrohlich und besorgniserregend. Es erfolgt daher oft eine umgehende ärztliche Vorstellung. Der erstbehandelnde Arzt ist gefordert, eine präzise, wegweisende Diagnostik mit den ihm verfügbaren Mitteln durchzuführen und anhand seiner erhobenen Befunde eine mögliche Notfallsituation zu erkennen, die eine umgehende weitere Abklärung notwendig macht.

Tabelle 1. Klassifikation von Schwindelbeschwerden nach der neuen Einteilung (ICVD) (mod. nach [8]). Entscheidend sind das zeitliche Auftreten, die Dauer und die Auslösbarkeit.

Akutes vestibuläres Syndrom (AVS)
- Permanenter Schwindel, Nausea, Gangunsicherheit, Bewegungs-
intoleranz
- Nystagmus
- DD: akute periphere Vestibulopathie, Stroke, MS, Komplikation
Otitis etc.
Episodisches vestibuläres Syndrom (EVS)
- Spontan (sEVS): keine Trigger, transient, meist <24h
- DD: M. Menière, vestibuläre Migräne, Arrhythmie, Panikattacke,
etc.
- Getriggert (tEVS): z. B. immer bei bestimmter Bewegung
- DD: BPLS, orthostatische Hypotonie, Chiari-Malformation,
cerebellärer Tumor, etc.
Chronisches vestibuläres Syndrom (CVS)
- Oszillopsien, Belastungsschwindel, Gangunsicherheit
DD: bilat. Vestibulopathie, Vestibularisschwannom, cerebelläre
Degeneration etc.

\section{Definition}

Ein neu aufgetretenes, akutes Ereignis mit konstantem Schwindel und dokumentierbarem Nystagmus wird definiert als akutes vestibuläres Syndrom (AVS) [1]. Hierbei handelt es sich häufig um eine akute periphere Vestibulopathie, auch Neuritis vestibularis genannt. Jedoch kann sich auch eine Ischämie oder eine Blutung im vertebrobasilären Stromgebiet ausschliesslich mit der Symptomatik eines AVS präsentieren. Etwa die Hälfte dieser Patienten zeigt initial keine weiteren fokal-neurologischen Zeichen [2]. Daher wird die Diagnose eines Hirnschlags bei gut einem Drittel dieser Fälle initial verpasst.

Rezidivierende Schwindelattacken und chronische Schwindel- bzw. Gleichgewichtsbeschwerden werden entsprechend in das episodische und chronische vestibuläre Syndrom eingeteilt (Tab. 1). Diese neue Einteilung ermöglicht eine erste Zuordnung der Symptomatik im Rahmen der Erstkonsultation und soll helfen, die weitere Diagnostik fokussiert voranzutreiben [3].

\section{Untersuchung/Abklärung}

Beim Erstkontakt ist neben der Schwindelanamnese die Erfragung allfälliger Begleitsymptome essenziell. Insbesonde-

$\begin{array}{ll}\text { Im Artikel verwendete Abkürzungen: } \\ \text { AVS } & \text { Akutes vestibuläres Syndrom } \\ \text { BPLS } & \text { Benigner paroxysmaler Lagerungsschwindel } \\ \text { CVS } & \text { Chronisches vestibuläres Syndrom } \\ \text { EVS } & \text { Episodisches vestibuläres Syndrom } \\ \text { HINTS } & \text { Head impulse test, Nystagmus, test of skew } \\ \text { KIT } & \text { Kopfimpulstest } \\ \text { SEVS } & \text { Spontanes episodisches vestibuläres Syndrom } \\ \text { tEVS } & \text { Getriggertes (engl.: triggered) episodisches vestibuläres } \\ & \text { Syndrom } \\ \text { VKIT } & \text { Video-Kopfimpulstest } \\ \text { VOR } & \text { Vestibulo-okulärer Reflex }\end{array}$


re Ohrsymptome wie Otalgie, eventuell mit Hörminderung, Tinnitus oder Otorrhö müssen an eine Affektion des Innenohrs denken lassen. Auch die Frage nach Kopfschmerzen oder sensomotorischen Defiziten sowie Doppelbildern oder Sprechschwierigkeiten gehören in die Anamnese.

Die klinische Untersuchung beim AVS konzentriert sich auf drei klinische Tests, die unter dem englischen Akronym HINTS (head impulse test, nystagmus, test of skew) zusammengefasst werden. Die diagnostische Wertigkeit der HINTS-Prüfung wurde bereits vielfach beschrieben, sodass sich diese in der neurootologischen Akutdiagnostik zunehmend etabliert hat. Anhand des dreiteiligen Tests - der Nystagmusprüfung, des Kopfimpulstests (KIT) und des test of skew (Prüfung der vertikalen Divergenz im Wechselabdecktest) - kann mit hoher Sicherheit zwischen einem zentralen Ereignis und einer akuten peripheren Vestibulopathie unterschieden werden [4]. Bei der Nystagmusprüfung mit und ohne Frenzelbrille liegt der Fokus auf der Richtung des Nystagmus (schnelle Phase) in Abhängigkeit von der Blickrichtung und sollte präzise dokumentiert werden (unidirektionaler Nystagmus: eher peripher; richtungswechselnder Nystagmus: zentral). Der KIT prüft den vestibulo-okulären Reflex (VOR), welcher der Blickstabilisierung dient. Hierbei wird der Kopf des Patienten durch den Untersucher impulsartig zur Seite rotiert, wobei der Patient gebeten wird, mit den Augen die Nasenspitze des Untersuchers zu fixieren. Ist der VOR ausgefallen, müssen die Augen nach einer Kopfbewegung zur betroffenen Seite das Sehziel refixieren, was anhand einer Korrektursakkade sichtbar wird. Ein pathologischer Kopfimpulstest bei unidirektionalem Nystagmus erhärtet die Diagnose eines peripher-vestibulären Ereignisses, wohingegen ein unauffälliger KIT trotz vorhandenem Nystagmus eine zentrale Genese hochwahrscheinlich macht. Beim test of skew sucht man eine vertikale Divergenz im Wechselabdecktest (skew deviation). Auch hier ist ein positiver Test hochprädiktiv für ein zentrales Ereignis, namentlich einen Hirnstamminfarkt. Tabelle 2 veranschaulicht die Interpretation der HINTS.

Bei Patienten mit AVS gehören zur weiteren klinischen Diagnostik der ORL- und Hirnnervenstatus, die Prüfung der Blickfolge und der Sakkaden, die Spitz-stumpf- und Kaltwarm-Diskrimination, sowie die üblichen Stand- und Gangprüfungen. Falls es der Allgemeinzustand erlaubt, wird in unserer Klinik üblicherweise auch ein Tonaudiogramm durchgeführt. Zudem haben wir den Video-Kopfimpulstest (vKIT) in die Akutdiagnostik beim AVS implementiert, der eine quantitative Messung des vestibulo-okulären Reflexes erlaubt. Er wird durch den diensthabenden Arzt ergänzend zur klinischen Untersuchung durchgeführt und dient der Diagnosesicherung, sowie zur Dokumentation des Ausmasses eines allfälligen peripher-vestibulären Defizits.

Falls sich in der Anamnese bereits ein episodisches vestibuläres Syndrom erheben lässt, das durch Lageänderungen ausgelöst wird und in Ruhe nicht vorhanden ist, kann man mit hoher Wahrscheinlichkeit von einem benignen paroxysmalen Lagerungsschwindel (BPLS) ausgehen. Die Lagerungsprüfungen für den posterioren und horizontalen Bogengang erlauben eine gezielte Identifizierung des betroffenen Bogenganges und damit eine hohe Heilungsrate durch die entsprechenden Repositionsmanöver. Differenzialdiagnostisch muss hierbei immer an einen zentralen Lageschwindel gedacht werden, da beispielsweise ein zerebellärer Tumor ähnliche Symptome auslösen kann. Daher ist bei untypischen oder prolongierten Befunden ein MRI zu erwägen.

\section{Haupt-Outcome der Erstkonsultation}

Bei der Erstkonsultation eines Schwindelpatienten sollte es gelingen, anhand seiner Angaben eine Zuordnung der Symptomatik in die beschriebenen Kategorien vorzunehmen. Wird ein akutes vestibuläres Syndrom identifiziert, werden die HINTS zur Differenzierung zwischen einem peripher-vestibulären Geschehen und einer zentralen Ursache herangezogen. Bei unklarer Situation helfen der erweiterte neurootologische Status, die Lagerungsprüfungen oder ein Hörtest weiter. Der vKIT kann eine Aussage über das Ausmass eines vestibulären Ausfalls machen oder aber einen intakten VOR bestätigen und somit bei gleichzeitigem Nystagmus die Indikation zur umgehenden neurologischen Abklärung und Behandlung erhärten [5]. Liegen anamnestische Hinweise oder Befunde (Otoskopie, Hörprüfung) für ein vorbestehendes Ohrleiden vor, insbesondere für eine akute oder chronische Otitis, muss an eine seltene, direkte Affektion des Innenohrs als Ursache des AVS gedacht werden. Die Zuweisung sollte dann umgehend $\mathrm{zu}$ einem Otologen oder in eine otologische Klinik und nicht in eine allgemeine Notfallstation erfolgen.

\section{Weiteres Vorgehen, Behandlung}

Weisen die HINTS bei einem Patienten mit AVS auf ein zentrales Ereignis hin, ist eine notfallmässige Zuweisung an ein Schlaganfallzentrum (stroke unit) empfohlen. Ein MRI gibt weitere Klarheit über Art und Ausmass der

Tabelle 2. Interpretation der HINTS bei einem AVS. Die drei Untersuchungen ergeben in Kombination eine Sensitivität von $100 \%$ und Spezifität von 96 \% zur Differenzierung zwischen einem peripher-vestibulären und einem zentralen Ereignis [1].

\begin{tabular}{c|ccccc} 
HINTS & Spontan-Nystagmus & & Kopfimpulstest & Skew deviation \\
Peripher & unidirektional & + & in die Richtung der langsamen Phase positiv & + & fehlt \\
Zentral & richtungswechselnder Nystagmus & $\begin{array}{l}\text { und/ } \\
\text { oder }\end{array}$ & in beide Richtungen negativ & $\begin{array}{l}\text { und/ } \\
\text { oder }\end{array}$ & vorhanden
\end{tabular}


Hirnaffektion. Bei sicherem Vorliegen einer akuten peripheren Vestibulopathie ohne Hinweise für ein Ohrleiden bedarf es keiner Bildgebung. Eine umfangreiche spezialärztliche und apparative Abklärung an unserer Klinik gibt einen klaren Überblick über das Ausmass der vestibulären Schädigung anhand der apparativen Testung der fünf Gleichgewichtsrezeptoren des Innenohrs (drei Bogengänge, Utriculus und Sacculus). Im Verlauf kann somit eine bessere Aussage über eine allfällige Erholung des Vestibularorgans gemacht werden. Therapeutisch stehen in diesem Fall die antivertiginöse Therapie, die Rehydrierung und die Gabe von systemischen Kortikosteroiden im Vordergrund. Letztere haben einen positiven Effekt auf den Heilungsprozess, insbesondere im Frühstadium [6]. Wir wenden hierfür unser Sechstageschema an: Dexamethason 40 mg Tag 1-3 und 10 mg Tag 4-6, falls keine Kontraindikationen vorliegen. Den grössten Einfluss auf den Krankheitsverlauf hat jedoch die vestibuläre Rehabilitation [7]. Der Patient sollte möglichst früh beginnen, das Gleichgewicht zu trainieren, allenfalls mit physiotherapeutischer Unterstützung. Eine gezielte vestibuläre Physiotherapie beschleunigt die zentrale Kompensation und damit die Rückkehr des Patienten in sein angestammtes Umfeld. Bei prolongierten Fällen oder unklaren Situationen bietet eine Anbindung an unser interdisziplinäres Zentrum für Schwindel und neurologische Sehstörungen eine differenzierte Abklärung und Behandlung der meist stark geplagten Patienten.

\section{Bibliografie}

1. Büki B, Straumann D, Tarnutzer A: Gleichgewichtsstörungen in der klinischen Praxis. 1. Aufl. Wien; Verlagshaus der Ärzte: 2008. 17-24.

2. Tarnutzer AA, Berkowitz AL, Robinson KA, Hsieh YH, NewmanToker DE: Does my dizzy patient have a stroke? A systematic review of bedside diagnosis in acute vestibular syndrome. CMAJ 2011; 183: E571-592.

3. Edlow JA, Newman-Toker D: Using the physical examination to diagnose patients with acute dizziness and vertigo. J Emerg Med 2016; 50:617-628.

4. Kattah JC, Talkad AV, Wang DZ, Hsieh YH, Newman-Toker DE: HINTS to diagnose stroke in the acute vestibular syndrome: three-step bedside oculomotor examination more sensitive than early MRI diffusion-weighted imaging. Stroke 2009; 40: 3504-3510.

5. Mantokoudis G, Tehrani AS, Wozniak A, et al.: VOR gain by head impulse video-oculography differentiates acute vestibular neuritis from stroke. Otol Neurotol 2015; 36: 457-465.

6. Strupp M, Magnusson M: Acute Unilateral Vestibulopathy. Neurol Clin 2015; 33: 669-685.

7. Whitney SL, Alghwiri AA, Alghadir A: An overview of vestibular rehabilitation. Handb Clin Neurol 2016; 137: 187-205.

8. Bisdorff AR, Staab JP, Newman-Toker DE: Overview of the International Classification of Vestibular Disorders. Neurol Clin 2015; 33: 541-550

Manuskript eingereicht: 4.11.2016

Manuskript nach Revision angenommen: 5.12.2016

Interessenskonflikt: Der Autor erklärt, dass kein Interessenskonflikt besteht.

\section{Dr. med. Vincent G. Wettstein}

Oberarzt

Klinik für Ohren-, Nasen-, Hals- und Gesichtschirurgie Interdisziplinäres Zentrum für Schwindel und neurologische Sehstörungen

Universitätsspital Zürich

Frauenklinikstrasse 24

8091 Zürich

vincent.wettstein@usz.ch 